\title{
Monocyte distribution width (MDW) performance as an early sepsis indicator in the emergency department: comparison with CRP and procalcitonin in a multicenter international European prospective study
}

Pierre Hausfater ${ }^{1,2,3^{*}} \mathbb{B}$, Neus Robert Boter ${ }^{4,5}$, Cristian Morales Indiano ${ }^{5,7}$, Marta Cancella de Abreu ${ }^{1,2}$, Adria Mendoza Marin 4,5, Julie Pernet ${ }^{1}$, Dolores Quesada ${ }^{5,8}$, Iris Castro ${ }^{9}$, Diana Careaga ${ }^{9}$, Michel Arock ${ }^{6}$, Liliana Tejidor ${ }^{9}$ and Laetitia Velly ${ }^{1,2}$

\begin{abstract}
Background: Early sepsis diagnosis has emerged as one of the main challenges in the emergency room. Measurement of sepsis biomarkers is largely used in current practice to improve the diagnosis accuracy. Monocyte distribution width (MDW) is a recent new sepsis biomarker, available as part of the complete blood count with differential. The objective was to evaluate the performance of MDW for the detection of sepsis in the emergency department (ED) and to compare to procalcitonin (PCT) and C-reactive protein (CRP).

Methods: Subjects whose initial evaluation included a complete blood count were enrolled consecutively in 2 EDs in France and Spain and categorized per Sepsis-2 and Sepsis-3 criteria. The performance of MDW for sepsis detection was compared to that of procalcitonin (PCT) and C-reactive protein (CRP).

Results: A total of 1,517 patients were analyzed: 837 men and 680 women, mean age $61 \pm 19$ years, 260 (17.1\%) categorized as Sepsis-2 and 144 patients (9.5\%) as Sepsis-3. The AUCs [95\% confidence interval] for the diagnosis of Sepsis-2 were 0.81 [0.78-0.84] and 0.86 [0.84-0.88] for MDW and MDW combined with WBC, respectively. For Sepsis-3, MDW performance was 0.82 [0.79-0.85]. The performance of MDW combined with WBC for Sepsis-2 in a subgroup of patients with low sepsis pretest probability was 0.90 [0.84-0.95]. The AUC for sepsis detection using MDW combined with WBC was similar to CRP alone $(0.85$ [0.83-0.87]) and exceeded that of PCT. Combining the biomarkers did not improve the AUC. Compared to normal MDW, abnormal MDW increased the odds of Sepsis-2 by factor of 5.5 [4.2-7.1, 95\% Cl] and Sepsis-3 by $7.6[5.1-11.3,95 \% \mathrm{Cl}]$.
\end{abstract}

Conclusions: MDW in combination with WBC has the diagnostic accuracy to detect sepsis, particularly when assessed in patients with lower pretest sepsis probability. We suggest the use of MDW as a systematic screening test, used together with qSOFA score to improve the accuracy of sepsis diagnosis in the emergency department.

*Correspondence: pierre.hausfater@aphp.fr

1 Emergency Department, Hôpital Pitié-Salpêtrière, APHP-Sorbonne Université, 83 Boulevard de l'hôpital, 75651 Paris Cedex 13, France

Full list of author information is available at the end of the article permits use, sharing, adaptation, distribution and reproduction in any medium or format, as long as you give appropriate credit to the original author(s) and the source, provide a link to the Creative Commons licence, and indicate if changes were made. The images or other third party material in this article are included in the article's Creative Commons licence, unless indicated otherwise in a credit line to the material. If material is not included in the article's Creative Commons licence and your intended use is not permitted by statutory regulation or exceeds the permitted use, you will need to obtain permission directly from the copyright holder. To view a copy of this licence, visit http://creativecommons.org/licenses/by/4.0/. The Creative Commons Public Domain Dedication waiver (http://creativeco mmons.org/publicdomain/zero/1.0/) applies to the data made available in this article, unless otherwise stated in a credit line to the data. 
Trial Registration ClinicalTrials.gov (NCT03588325).

Keywords: Monocyte volume distribution width, MDW, Procalcitonin, C-reactive protein, Emergency department, Sepsis

\section{Introduction}

A significant proportion of patients developing sepsis enter the health system through an emergency department (ED) [1-4]. Because the surviving sepsis campaign promotes targeting short-term specific, goal-directed therapy bundles, early sepsis diagnosis has therefore emerged as one of the main challenges for emergency physicians [5]. Although the systemic inflammatory response syndrome (SIRS) or quick Sequential Organ Failure Assessment (qSOFA) score are helpful clinical tools for sepsis suspicion during early triage, sepsis diagnosis may still be delayed or misdiagnosed $[4,6]$. So, measurement of sepsis biomarkers is largely used in current practice to improve the diagnosis accuracy [7].

MDW, a hematologic parameter measured as part of the complete blood count with differential (CBC-DIFF) and describing the size distribution of circulating monocytes, was shown in two recent North American studies $[8-10]$ to be a valuable new sepsis biomarker for the early detection of patients in the emergency department. However, little is known about the performance of MDW and how it compares to those of the most frequently used sepsis biomarkers: C-reactive protein (CRP) and procalcitonin (PCT) [11].

The primary objective of this study was to confirm the clinical validity and performance of MDW to identify patients who developed sepsis in a European ED population. Secondary objectives were to compare the performance of MDW with CRP and PCT and to verify MDW cutoff for tripotassium ethylenediaminetetraacetic acid $\left(\mathrm{K}_{3} \mathrm{EDTA}\right)$ tubes.

\section{Methods}

This was an international, blinded, prospective cohort study enrolling patients presenting to two adult large EDs at the Pitié-Salpêtrière APHP-Sorbonne Université hospital in Paris (LPS), France, and the Hospital Universitari Germans Trias i Pujol in Badalona, Spain (UHG). The study assessed MDW's ability to detect the development of sepsis in consecutive adult patients (18-89 years) presenting to the ED and who had a CBC-DIFF ordered. The study was registered in ClinicalTrials.gov (NCT03588325) and approved by the ethical committees of the respective participating countries.

\section{Patients}

The study prospectively enrolled consenting adults (over 18-year-old) presenting to the ED, whose initial evaluation included a CBC-DIFF. Exclusion criterion is as shown in Fig. 1. Emergency physicians were free to order necessary tests according to the standard of care. Treating physicians were blinded to MDW, PCT and CRP values obtained by protocol, but received PCT and/or CRP results they ordered as current practice.

Both PCT and CRP are used routinely at LPS, and CRP is used routinely at UHG.

\section{Blood sampling}

Briefly, patients who were identified to have a CBC-DIFF ordered by the physician were asked to participate. After informed consent, baseline blood draws were performed. An additional $\mathrm{K}_{3}$ EDTA tube was drawn together with a sample for PCT and CRP measurement as well as routine blood tests at the discretion of the treating physician. Samples for MDW measurement were analyzed on a UniCel DxH 900 analyzer (Beckman Coulter, Inc., Brea, CA) with version 1.0.0.329 software within two hours of collection. This instrument measures specific cell volume variables and the distribution of cell volumes within a group of white blood cells (WBC). Quality control was performed daily with COULTER 6C Plus Cell Control to monitor the DxH 900 system performance. COULTER LATRON CP-X Control was used as part of the daily quality control procedure to monitor volume, conductivity and light scatter measurements. PCT and CRP concentrations were measured on Cobas analyzer (Roche Diagnostics, Meylan, France) at LPS, while UHG utilized Liaison XL (DiaSorin, Saluggia, Italy) and AU5800 (Beckman Coulter, Inc, Brea, CA, USA) analyzers for PCT and CRP measurements, respectively.

\section{Clinical data, follow-up, blinding and adjudication}

Clinical data at presentation, including past medical history, assessment of vital signs, symptoms, SIRS criteria [12], qSOFA [13] and SOFA scores [14], microbiological testing and treatments were recorded on an electronic case report form and patients followed up for at least $12 \mathrm{~h}$. The clinical research team and physician adjudicators were blinded to MDW results at the time of clinical data entry and during assignment of the patients to 


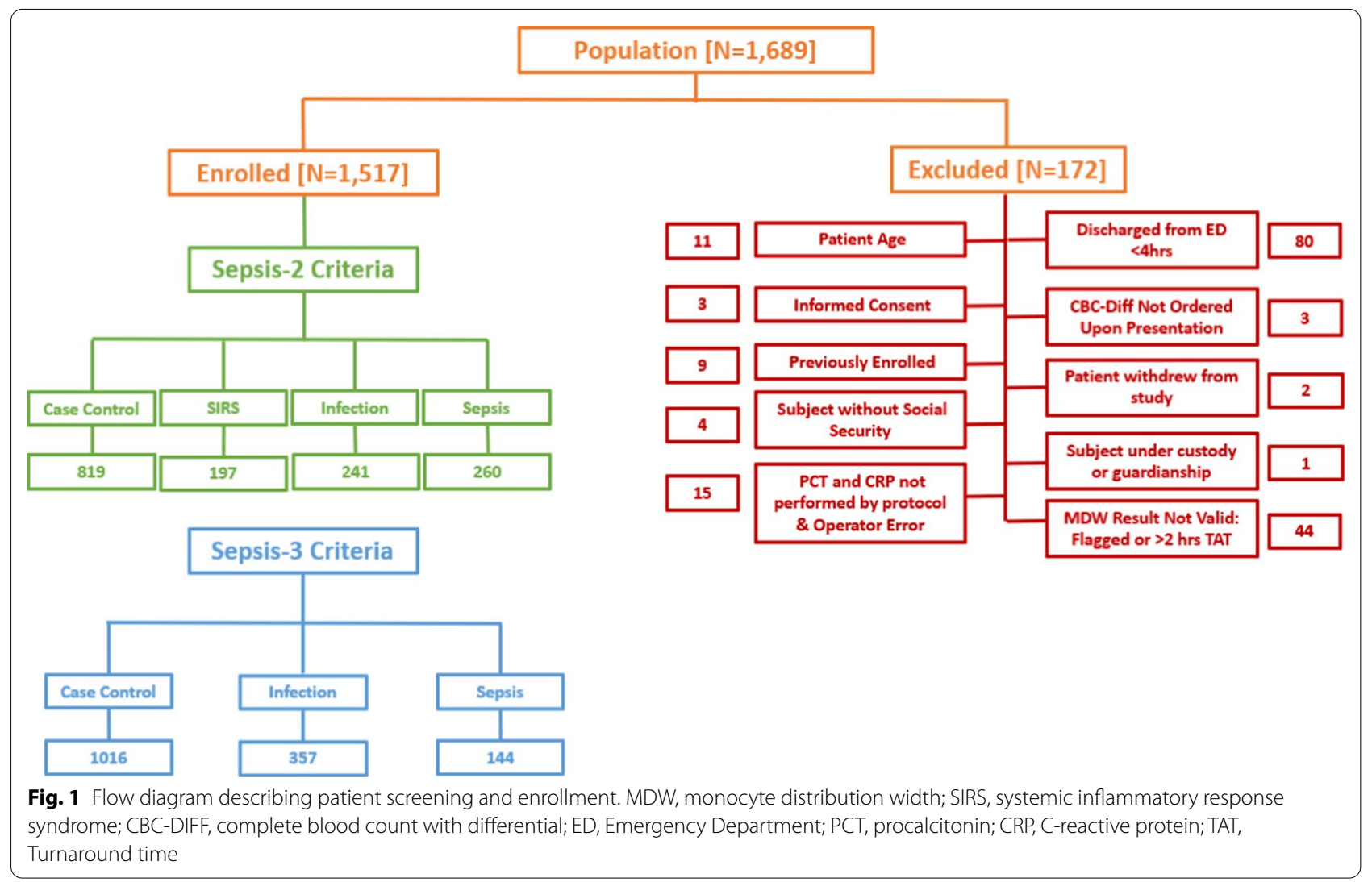

a clinical category. Adjudicators were also blinded to the results of PCT and CRP if not ordered by the treating ED physician.

Study subjects were classified by at least two independent physician adjudicators at each site. Discordances were arbitrated by a third independent physician. Adjudicators categorized subjects based on the "Sepsis-2" consensus criteria [15], such as non-SIRS or case controls (zero or one SIRS criterion and no infection), SIRS ( $\geq 2$ SIRS criteria and no infection), infection (suspected or confirmed infection with $0-1$ SIRS criteria), sepsis (infection plus $\geq 2$ SIRS) (including sepsis [no organ failures], severe sepsis [sepsis with one or more organ failures] and septic shock [sepsis with refractory hypotension]). Adjudicated categories per Sepsis-3 criteria [16] included controls, infection and sepsis (based on SOFA score criteria). In order to characterize sepsis as being present upon ED admission, sepsis criteria had to be fulfilled within $12 \mathrm{~h}$ of the initial CBC-DIFF in patients with suspected infection (as reflected by initiation of diagnostic infection workup) and adjudication was based on the retrospective chart review of tests ordered and clinical data available within the first $12 \mathrm{~h}$ of ED presentation. If no infection work-up was performed within $12 \mathrm{~h}$, or if the adjudicator believed that the infection work-up showed no evidence of infection, the patient was categorized as "not infected" or SIRS by the adjudicator. The test results were extracted from the medical records 7-10 days later, including cultures, molecular tests (e.g., polymerase chain reaction), antigens tests (immunoassay) and relevant imaging. A subgroup, consisting of subjects for whom no CRP or PCT was ordered by the emergency physician, was identified (post study) and defined as having low sepsis pretest probability.

\section{Statistical analysis}

General descriptive statistics and box plots were calculated for cell population variables. The sample size calculations were based on 95\% two-sided confidence interval and $80 \%$ power. Proc power of SAS 9.3 was used for calculating sample size based on the approach presented in Johnson et al. [17] A target sensitivity of $75 \%$ with the lower limit of the $95 \%$ two-sided confidence interval of $65 \%$ and a target specificity of $70 \%$ with the lower limit of the 95\% two-sided confidence interval of $65 \%$ were assumed. A minimum of 189 septic subjects and a minimum of 817 non-septic subjects were needed from both sites combined.

Diagnostic ability was evaluated in terms of the area under the ROC curve (AUC), sensitivity, specificity, 
positive predictive value (PPV), negative predictive value (NPV), positive and negative likelihood ratios, along with their 95\% confidence intervals (CIs). The score approach was used to calculate CI for sensitivity, specificity, PPV and NPV. Differences in AUC were used to demonstrate the added value of MDW in comparison with WBC alone, calculated using a one-predictor variable logistic model with WBC and a two-predictor variables logistic model with both WBC and MDW, as the predictor, and using sepsis status as the response. A similar approach was applied to analyze the diagnostic ability of PCT and CRP, and the combination with WBC and MDW for sepsis detection. AUC comparisons along with their $\mathrm{CI}$ were calculated as described by DeLong et al. [18] SAS 9.4 (SAS Institute, Cary, NC) statistical program was used for data analyses.

\section{Cutoff determination}

PCT cutoff of $>0.25 \mu \mathrm{g} / \mathrm{mL}$ was based on the literature. The determination of the cutoff for CRP $(>22 \mathrm{mg} / \mathrm{L}$ ) was based on the Youden index using the data from this clinical study. The proposed MDW cutoff of 21.5 units for $\mathrm{K}_{3}$ EDTA was based on previously established MDW cutoff (in $\mathrm{K}_{2}$ EDTA) for sepsis identification in the ED and internal testing demonstrating a shift of 1.5 units for blood specimens collected in $K_{3}$ EDTA versus $K_{2}$ EDTA (data not shown). This study validates the new MDW cutoff of 21.5 for $\mathrm{K}_{3}$ EDTA.

\section{Probabilities and odds ratios}

Identification of sepsis in the ED relies on the physician's ability to assess probability of disease based on presenting symptoms. To reflect this approach, we analyzed the probability and likelihood ratios for sepsis based upon the values of MDW in combination with SIRS or qSOFA parameters determined during the initial patient encounter in the ED (typically within the first $2 \mathrm{~h}$ of ED admission). Predicted probability of a positive sepsis diagnosis was calculated from the positive likelihood ratios (LRs + ) as previously described $[19,20]$. In this approach, predicted sepsis probability after receiving test results, or posttest probability $P_{1}$, is based on an estimated pretest probability $P_{0}$ and LR + and is calculated as:

$$
P_{1}=\frac{P_{1} \times \mathrm{LR}+}{\left(1-P_{0}+P_{0} \times \mathrm{LR}+\right)}
$$

where $P_{0}$ is the sepsis prevalence of the study cohort. The odds ratios (ratios of posttest probabilities) for sepsis diagnosis between parameter combinations with abnormal and normal MDW values were calculated based on prevalence of sepsis-2 or sepsis-3 for the study population.

\section{Results}

As shown in the flow diagram of the study (Fig. 1), from August 2, 2018, through June 27, 2019, 1,689 patients were screened and enrolled, and 172 were excluded due to patient not meeting inclusion criteria, inadequate

Table 1 Demographic data. Data were available for all the patients, unless indicated by N. Quantitative data are expressed as Medians (interquartile range)

\begin{tabular}{|c|c|c|c|c|c|}
\hline & LPS & UHG & Sites combined & $\begin{array}{l}\text { Sepsis-2 } \\
N=260\end{array}$ & $\begin{array}{l}\text { Sepsis-3 } \\
N=144\end{array}$ \\
\hline \multicolumn{6}{|l|}{ Clinical characteristics } \\
\hline Female (No. \%) & $404(48.3 \%)$ & $276(40.6 \%)$ & $680(44.8 \%)$ & $101(38.9 \%)$ & $46(31.9 \%)$ \\
\hline Male (No. \%) & $433(51.7 \%)$ & $404(59.4 \%)$ & $837(55.2 \%)$ & $159(61.2 \%)$ & $98(68.1 \%)$ \\
\hline Age, years & $57(39-71)$ & $72(60-80)$ & $64(47-76)$ & $66(51-77)$ & $72(61-80)$ \\
\hline Temperature $\left({ }^{\circ} \mathrm{C}\right) \mathrm{N}=1506$ & $36.6(36.2-37.0)$ & $36.2(36.0-36.7)$ & $36.5(36.0-36.9)$ & $37.1(36.5-38.2)$ & $36.8(36.2-38.0)$ \\
\hline Heart rate (beats/min) & $85(73-100)$ & $82(70-98)$ & $84(71-99)$ & $100(92-110)$ & $93(75-106)$ \\
\hline Respiratory (breaths/min) N=865 & $20(16-22)$ & $18(16-24)$ & $19.0(16-24)$ & $22(18-26)$ & $23(18-26)$ \\
\hline Immunosuppression & $38(4.5 \%)$ & $101(14.9 \%)$ & 139 (9.2\%) & $38(14.6 \%)$ & $22(15.3 \%)$ \\
\hline \multicolumn{6}{|l|}{ Laboratory parameters } \\
\hline $\mathrm{WBC}\left(1 \times 10^{3} / \mu \mathrm{L}\right)$ & $8.33(6.24-10.94)$ & $9.17(6.86-11.89)$ & $8.71(6.50-11.34)$ & $13.02(9.02-16.28)$ & $10.82(8.27-15.03)$ \\
\hline $\operatorname{PMN}\left(1 \times 10^{3} / \mu \mathrm{L}\right)$ & $5.80(3.88-8.44)$ & $6.74(4.73-9.42)$ & $6.27(4.23-8.90)$ & $10.65(7.09-14.04)$ & $8.99(6.15-13.18)$ \\
\hline $\mathrm{EO}\left(1 \times 10^{3} / \mu \mathrm{L}\right)$ & $0.07(0.02-0.15)$ & $0.07(0.02-0.14)$ & $0.07(0.02-0.14)$ & $0.02(0.01-0.06)$ & $0.02(0.0-0.06)$ \\
\hline MDW (U) & $19.28(17.85-21.68)$ & 21.09 (19.19-24.03) & 20.15 (18.22-22.79) & $23.92(21.54-26.94)$ & $24.61(22.33-28.47)$ \\
\hline PCT (ng/mL) & $0.04(0.02-0.10)$ & $0.06(0.02-0.24)$ & $0.05(0.02-0.15)$ & $0.21(0.07-0.79)$ & $0.34(0.13-1.54)$ \\
\hline CRP (mg/L) & $5.22(0.85-31.76)$ & $18.30(4.75-82.50)$ & $10.10(1.91-52.89)$ & 82.50 (36.90-185.50) & 95.35 (44.13-197.98) \\
\hline $\operatorname{CREAT}(\mu \mathrm{mol} / \mathrm{L}) \mathrm{N}=1489$ & $76.00(64.00-95.00)$ & $80.40(62.80-113.20)$ & $78.00(63.60-102.00)$ & $84.00(65.40-123.00)$ & 115.35 (74.30-186.50) \\
\hline
\end{tabular}

LPS, Pitié-Salpêtrière APHP-Sorbonne Université Hospital; UHG, Hospital Universitari Germans Trias i Pujol; WBC, white blood count; PMN, polymorphonuclear; EO, eosinophils; MDW, monocyte distribution width; PCT, procalcitonin; CRP, C-reactive protein; CREAT, creatinine 
sample collection or screening errors. The final analysis included 1,517 patients (LPS 837, UHG 680). Demographic features are shown in Table 1 . There were 837 men and 680 women, median age 64 (IQ 47-76 years). Using Sepsis-2 criteria, 819 patients (54\%) were categorized as non-SIRS/non-sepsis (case controls) while 260 (17.1\%) had sepsis, including 38 with severe sepsis. According to Sepsis-3 criteria, 1,016 patients (67\%) were adjudicated as case controls and 144 patients (9.5\%) had sepsis. Eighty-five percent of Sepsis-2 patients had at least one microbiological test performed, of which 58\% were positive. Bacterial cultures grew with Gram-positive species in 16 patients, Gram-negative in 62 patients and mixed gram stain in 10 . These cultures were 41 from urine, 24 from blood, 14 from sputum and 9 from other sources (Additional file 2: Sup. Table 5). Five hundred and fifty-three patients (36.5\%) fell in the subgroup of the low pretest probability of sepsis (no CRP or PCT ordered by the emergency physicians).

\section{MDW performance for sepsis detection}

Figure 2A, B illustrates box whisker plots for a single baseline MDW measurement according to Sepsis-2 (A) or Sepsis-3 (B) criteria showing increases in MDW values with infection and sepsis severity, regardless of sepsis criteria used. In $\mathrm{K}_{3}$ EDTA tubes, the MDW cutoff of 21.5 provides an optimum diagnostic power by balancing the ability to detect positive sepsis patients (sensitivity) and case controls (specificity). The area under the receiver operating characteristic (AUROC) curves for Sepsis-2 was 0.81 [95\% confidence interval 0.78-0.84] for MDW vs 0.76 [0.72-0.79] for WBC and 0.86 [0.840.88 ] when combined with WBC (Fig. 3a). For Sepsis-3, MDW AUROC was 0.82 [0.79-0.85] compared to 0.65 [0.60-0.70] for WBC (Fig. 3b). Adding WBC to MDW assessment added no value for Sepsis-3 diagnosis. For the subgroup of patients with low sepsis pretest probability, AUROC for Sepsis-2 was 0.83 [0.75-0.91] for MDW alone and 0.90 [0.84-0.95] when combined with WBC (Fig. 3c).

\section{MDW added value for sepsis detection}

The value of MDW significantly modified the observed posttest probability of sepsis vs. the baseline probability regardless of the sepsis definitions, specific clinical risk scores (SIRS and qSOFA) and values of WBC, with abnormal (>21.5) MDW increasing and normal MDW decreasing the posttest probability. Compared to normal MDW, abnormal MDW increased the odds of Sepsis-2 by a factor of 5.5 [4.2-7.1, 95\% CI] and the odds of Sepsis-3 by $7.6[5.1-11.3,95 \% \mathrm{CI}]$ (Table 2), with highest increases occurring in patients with low SIRS and qSOFA scores (Table 2 and Additional file 3: Sup. Figure 6). Abnormal
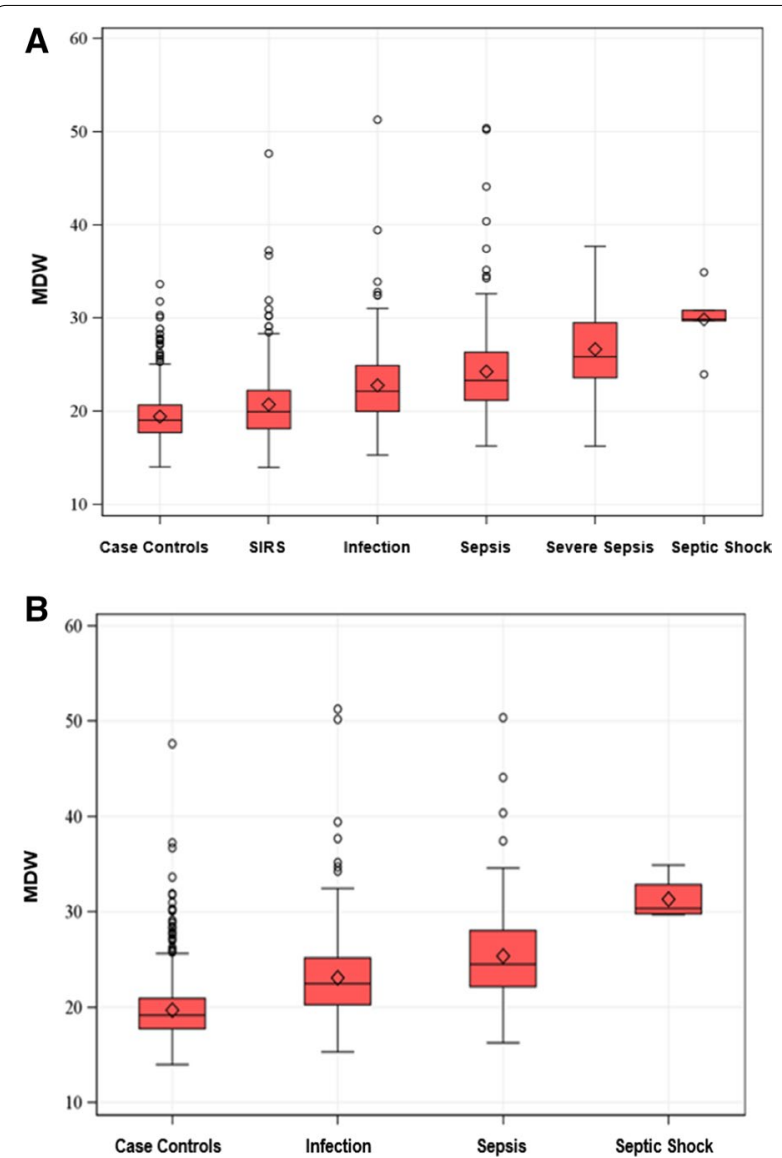

Fig. 2 Box plots of MDW baseline values conforming to sepsis classification by Sepsis-2 criteria (A) and Sepsis-3 criteria (B). MDW, monocyte distribution width; SIRS, systemic inflammatory response syndrome

MDW increased the probability of Sepsis-2 for all ranges of WBC including in patients with low pretest probability with similar effect observed using Sepsis-3 adjudication (Fig. 4a-d and Additional file 4: Sup. Figure 7).

\section{Comparison of MDW with PCT and CRP performance for sepsis detection}

The respective performance of PCT and CRP and combined with WBC and MDW is represented in Table 3. Overall, the diagnostic ability of MDW combined with WBC was similar to CRP alone (AUROC: 0.86 [0.840.88 ] vs. 0.85 [0.83-0.87], respectively) and performed better than PCT alone (AUROC: 0.78 [0.75-0.81]). Adding either CRP or PCT to MDW/WBC analysis did not improve the AUC. The sensitivity and specificity of using MDW and/or WBC in combination, based on their individual cutoffs, is shown in Additional file 1: Sup. Table 4. If either parameter was abnormal ("or") the sensitivity was $>90 \%$, if both parameters were abnormal ("and") 

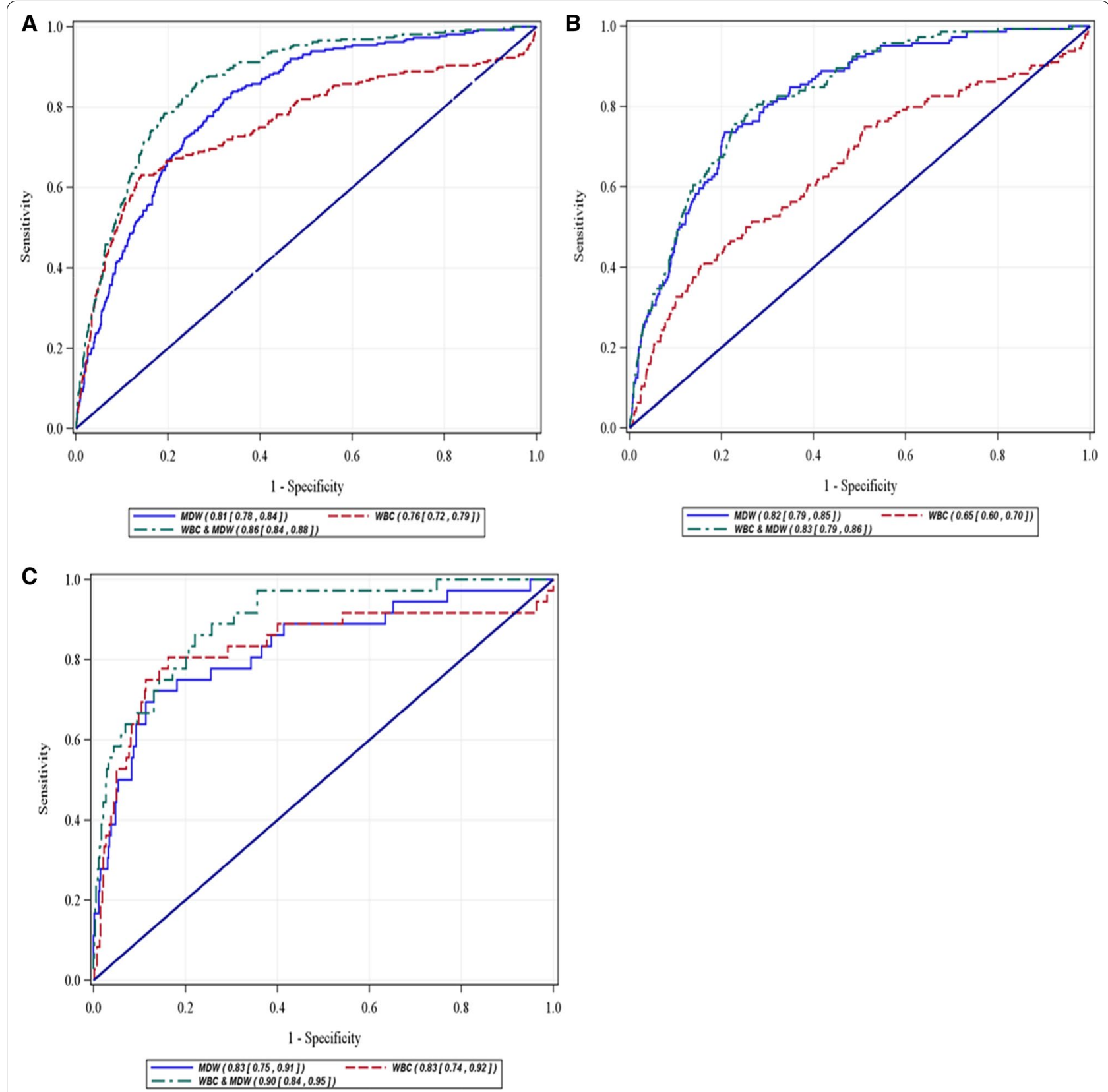

Fig. 3 MDW, WBC and MDW + WBC performance for sepsis diagnosis. A Sepsis-2. B Sepsis-3. C Sepsis-2, low pretest probability population (CRP or PCT not ordered by emergency physician). MDW, monocyte distribution width; WBC, white blood count

then as expected the specificity of the combination of MDW + WBC was high (>90\%).

Figure $5 \mathrm{a}-\mathrm{d}$ represents the posttest sepsis probabilities of the sequential results of baseline WBC, MDW and PCT or CRP tests at their specified cutoffs. When WBC is normal (in the $4,000-12,000 / \mathrm{mm}^{3}$ range), an MDW value $>21.5$ is associated with a tenfold increase of Sepsis-2 probability (compared to MDW normal), and an eightfold increase of Sepsis-3. Similarly, when WBC is abnormal $\left(<4,000\right.$ or $\left.>12,000 / \mathrm{mm}^{3}\right)$ and $\mathrm{MDW}>21.5$, the probability of Sepsis-2 is twice the one when MDW is normal, and sevenfold for Sepsis-3.

When WBC is normal but MDW is abnormal ( $>21.5$ ), addition of PCT increases probability of sepsis to the same level as when both (WBC, MDW) parameters were abnormal, threefold above the pretest probability (see Fig. $5 \mathrm{~b}, \mathrm{PCT}>0.25$ and $\mathrm{MDW}>21.5$ 
Table 2 Added value of MDW to SIRS criteria for Sepsis-2 diagnosis (pretest=0.17), and to qSOFA for Sepsis-3 diagnosis (pretest=0.9)

\begin{tabular}{|c|c|c|c|c|c|c|}
\hline Sepsis 2 \& SIRS & No SIRS & 1 SIRS & 2 SIRS & 3 SIRS & 4 SIRS & Total \\
\hline Total patients & 515 & 594 & 271 & 117 & 20 & 1517 \\
\hline Sepsis patients (\#) & 4 & 21 & 141 & 81 & 13 & 260 \\
\hline Sepsis probability, MDW unknown & $0.8 \%$ & $3.5 \%$ & $51.8 \%$ & $69.0 \%$ & $64.8 \%$ & $17.0 \%$ \\
\hline Sepsis probability, MDW normal & $0.3 \%$ & $1.7 \%$ & $31.1 \%$ & $37.6 \%$ & $28.4 \%$ & $6.6 \%$ \\
\hline Sepsis probability, MDW abnormal & $2.4 \%$ & $7.8 \%$ & $71.2 \%$ & $83.6 \%$ & $84.5 \%$ & $36.1 \%$ \\
\hline Sepsis odds (MDW abnormal/normal) [95\% Cl] & 9.3 & 4.7 & 2.3 & 2.2 & 3.0 & $5.5[4.2-7.1]$ \\
\hline Sepsis 3 \& qSOFA & No qSOFA & 1 qSOFA & 2 qSOFA & 3 qSOFA & & Total \\
\hline Total patients & 1136 & 347 & 33 & 1 & & 1517 \\
\hline Sepsis patients (\#) & 53 & 72 & 18 & 1 & & 144 \\
\hline Sepsis probability, MDW unknown & $4.4 \%$ & $19.8 \%$ & $53.1 \%$ & & & $9.0 \%$ \\
\hline Sepsis probability, MDW normal & $1.2 \%$ & $8.1 \%$ & $28.8 \%$ & & & $2.7 \%$ \\
\hline Sepsis probability, MDW abnormal & $12.0 \%$ & $31.9 \%$ & $63.9 \%$ & & & $20.6 \%$ \\
\hline Sepsis odds (MDW abnormal/normal) [95\% Cl] & 10.1 & 3.9 & 2.2 & & & $7.6[5.1-11.3]$ \\
\hline
\end{tabular}

MDW, monocyte distribution width; SIRS, systemic inflammatory response syndrome; qSOFA, quick Sequential Organ Failure Assessment; CI, Confidence Interval

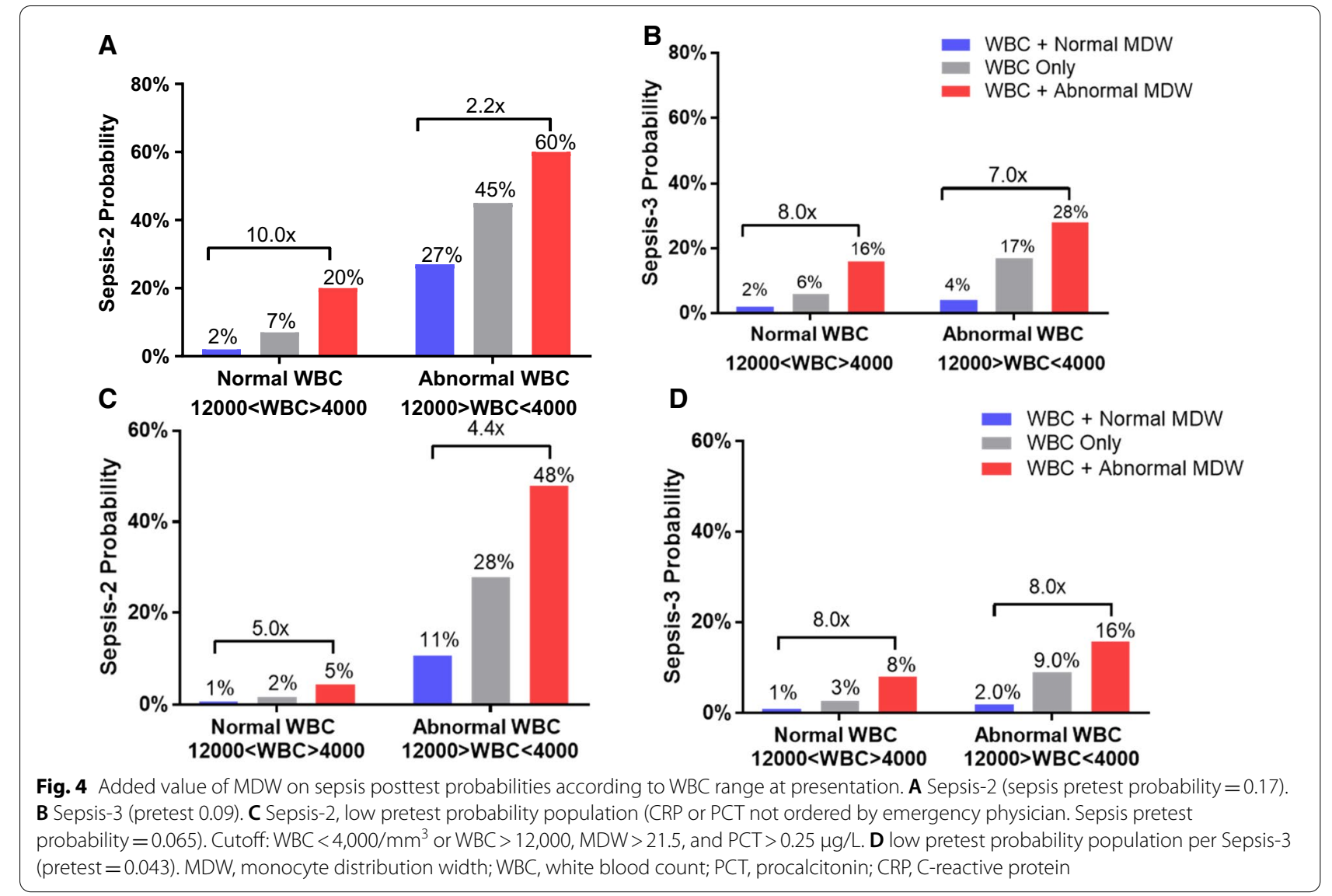

and WBC normal sepsis probability is $30 \%$ ). A similar pattern was observed with CRP. Similarly, when WBC is abnormal but MDW is normal, addition of
$\mathrm{PCT}>0.25 \mu \mathrm{g} / \mathrm{L}$ increases the Sepsis-2 probability approximately threefold comparable to when both WBC and MDW are both abnormal. 
Table 3 Comparison of MDW with WBC, PCT and CRP performance for sepsis detection Cutoff: WBC $<4,000 / \mathrm{mm}^{3}$ or WBC $>12,000$ / $\left.\mathrm{mm}^{3}, \mathrm{MDW}>21.5, \mathrm{PCT}>0.25 \mu \mathrm{g} / \mathrm{L}, \mathrm{CRP}>22 \mathrm{mg} / \mathrm{L}\right)$

\begin{tabular}{|c|c|c|c|c|c|c|c|}
\hline \multicolumn{8}{|l|}{ Sepsis-2 } \\
\hline Parameter & $\begin{array}{l}\text { Sensitivity } \\
{[95 \% \mathrm{Cl}]}\end{array}$ & $\begin{array}{l}\text { Specificity } \\
{[95 \% \mathrm{Cl}]}\end{array}$ & PPV [95\%Cl] & NPV $[95 \% \mathrm{Cl}]$ & $\mathrm{LR}+[95 \% \mathrm{Cl}]$ & $\mathrm{LR}-[95 \% \mathrm{Cl}]$ & AUC $[95 \% \mathrm{Cl}]$ \\
\hline MDW & $0.75[0.69-0.80]$ & $0.73[0.70-0.75]$ & $0.36[0.32-0.40]$ & $0.93[0.92-0.95]$ & $2.76[2.46-3.09]$ & $0.34[0.28-0.43]$ & 0.81 [0.78-0.84] \\
\hline WBC & $0.69[0.63-0.74]$ & $0.83[0.81-0.85]$ & $0.45[0.40-0.50]$ & $0.93[0.91-0.94]$ & $4.00[3.50-4.60]$ & $0.40[0.30-0.50]$ & $0.76[0.72-0.79]$ \\
\hline PCT & $0.45[0.39-0.51]$ & $0.88[0.86-0.90]$ & $0.44[0.38-0.50]$ & $0.89[0.87-0.90]$ & 3.80 [3.10-4.65-] & $0.62[0.56-0.70]$ & $0.78[0.75-0.81]$ \\
\hline CRP & 0.85 [0.80-0.88] & $0.72[0.70-0.75]$ & $0.39[0.35-0.43]$ & $0.96[0.94-0.97]$ & 3.06 [2.76-3.39] & $0.21[0.16-0.28]$ & 0.85 [0.83-0.87] \\
\hline$M D W+W B C$ & & & & & & & $0.86[0.84-0.88]$ \\
\hline $\mathrm{MDW}+\mathrm{PCT}$ & & & & & & & $0.81[0.78-0.84]$ \\
\hline $\mathrm{MDW}+\mathrm{CRP}$ & & & & & & & $0.85[0.82-0.87]$ \\
\hline$M D W+W B C+P C T$ & & & & & & & $0.86[0.84-0.89]$ \\
\hline $\mathrm{MDW}+\mathrm{WBC}+\mathrm{CRP}$ & & & & & & & $0.87[0.85-0.89]$ \\
\hline \multicolumn{8}{|l|}{ Sepsis-3 } \\
\hline MDW & $0.81[0.73-0.86]$ & $0.69[0.67-0.72]$ & $0.22[0.18-0.25]$ & $0.97[0.96-0.98]$ & $2.63[2.35-2.94]$ & $0.28[0.20-0.39]$ & 0.82 [0.79-0.85] \\
\hline WBC & $0.49[0.41-0.57]$ & $0.77[0.74-0.79]$ & $0.18[0.15-0.22]$ & $0.94[0.92-0.95]$ & $2.10[1.70-2.50]$ & $0.70[0.60-0.80]$ & $0.65[0.60-0.70]$ \\
\hline $\mathrm{PCT}$ & $0.60[0.52-0.68]$ & $0.87[0.85-0.89]$ & $0.33[0.27-0.39]$ & $0.95[0.94-0.96]$ & $4.63[3.83-5.60]$ & $0.46[0.37-0.56]$ & $0.84[0.80-0.87]$ \\
\hline CRP & 0.89 [0.83-0.93] & $0.68[0.66-0.71]$ & $0.23[0.19-0.26]$ & 0.98 [0.97-0.99] & $2.79[2.53-3.07]$ & $0.16[0.10-0.26]$ & $0.85[0.82-0.87]$ \\
\hline$M D W+W B C$ & & & & & & & 0.83 [0.79-0.86] \\
\hline$M D W+P C T$ & & & & & & & 0.82 [0.79-0.86] \\
\hline $\mathrm{MDW}+\mathrm{CRP}$ & & & & & & & $0.85[0.82-0.88]$ \\
\hline$M D W+W B C+P C T$ & & & & & & & $0.83[0.80-0.86]$ \\
\hline$M D W+W B C+C R P$ & & & & & & & $0.85[0.82-0.87]$ \\
\hline
\end{tabular}

MDW, monocyte distribution width; WBC, white blood count; PCT, procalcitonin; CRP, C-reactive protein; PPV, positive predictive value; NPV, negative predictive value; $\mathrm{LR}+$ : positive likelihood ratio. $\mathrm{LR}$-, negative likelihood ratio; $\mathrm{AUC}$ : area under the $\mathrm{ROC}$ curve; $\mathrm{Cl}$ confidence interval

\section{Discussion}

In this multicenter clinical trial conducted in two large European EDs, we confirmed the performance of MDW as an early sepsis biomarker with an AUC of 0.81 [0.78$0.84]$ and 0.86 [0.84-0.88] when combined with WBC for Sepsis-2 criteria. These results are in accordance with those of two recent North American studies which reported very similar performance of MDW (AUC 0.79 [0.73-0.84] and 0.79 [0.76-0.82], respectively) $[8,9]$ which argues for the robustness and reproducibility of this new biomarker in different populations. At an optimal cutoff of 21.5 in $\mathrm{K}_{3}$ EDTA tubes, a sensitivity of $75 \%$ $[69-80,95 \% \mathrm{CI}]$ and specificity of $73 \%[70-75,95 \% \mathrm{CI}]$ were observed which again agrees well with previous studies in North America and demonstrates that the anticoagulant does not affect the diagnostic accuracy of the biomarker. Moreover, we reported that MDW combined with WBC was particularly useful to detect sepsis in a low pretest probability subgroup of patients (AUC: 0.90 [0.84-0.95]), that is patients for whom no sepsis biomarker was ordered by the emergency physicians upon presentation. MDW may be displayed routinely as part of $\mathrm{CBC}$ with differential for most of the patients who have blood drawn in the ED and thus provides an added value to the early clinical evaluation. Indeed, early sepsis detection is warranted to initiate specific goal-directed therapy bundles [5]; hence, the systematic SIRS criteria and qSOFA score calculation are highly recommended. However, both SIRS and qSOFA lack sensitivity and specificity and may falsely lead to over or under suspicion of sepsis $[4,6,21,22]$. In the present study, we confirmed that MDW provided an added diagnostic power to SIRS and qSOFA variables, with an overall odd of 5.5 and 7.6 for Sepsis-2 and Sepsis-3, respectively, when MDW $>21.5$ (Table 2 and Fig. 5). As recently published by Crouser, et al., because MDW value can be displayed with $\mathrm{CBC}$ results early in the ED's course of the patient, it may be considered as a fifth SIRS or fourth qSOFA criteria [10].

MDW combined with WBC had a similar AUC compared to CRP $(0.85$ [0.82-0.87]) and performed better than PCT (0.78 [0.75-0.81]) (Table 3). To our knowledge, this was the first report of the comparison between MDW, PCT and CRP in the emergency department, in contrast to the study by Polilli, et al., who reported nearly overlapping AUC for MDW and PCT (0.87 vs. 0.88 , respectively) in a cohort of hospitalized patients for suspected infection or sepsis [11]. The performances of PCT and CRP we observed were much higher than what 

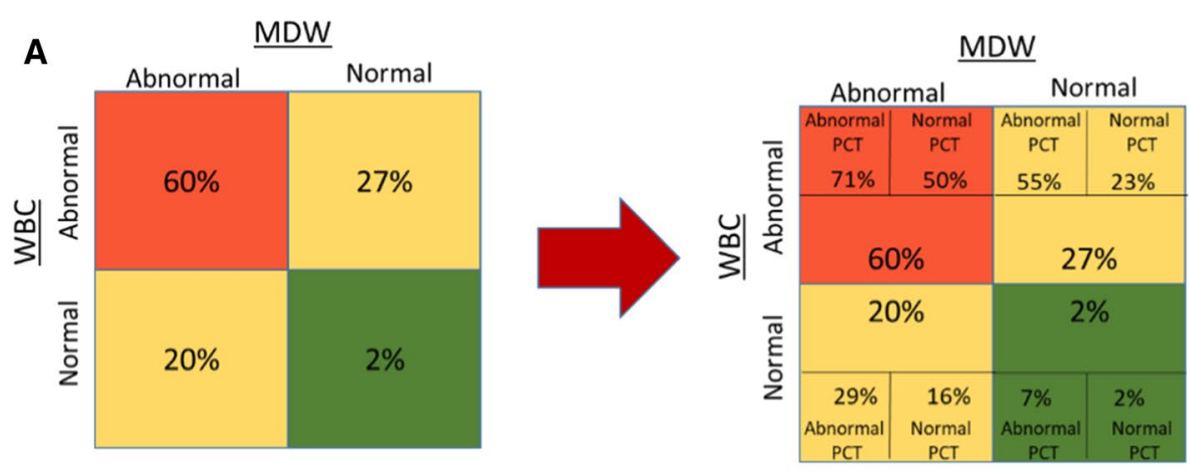

B

MDW
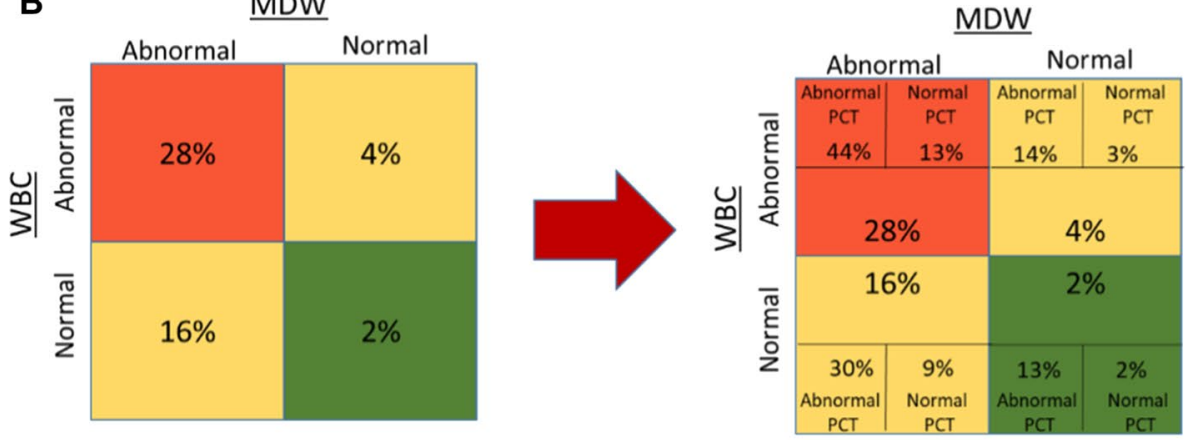

C
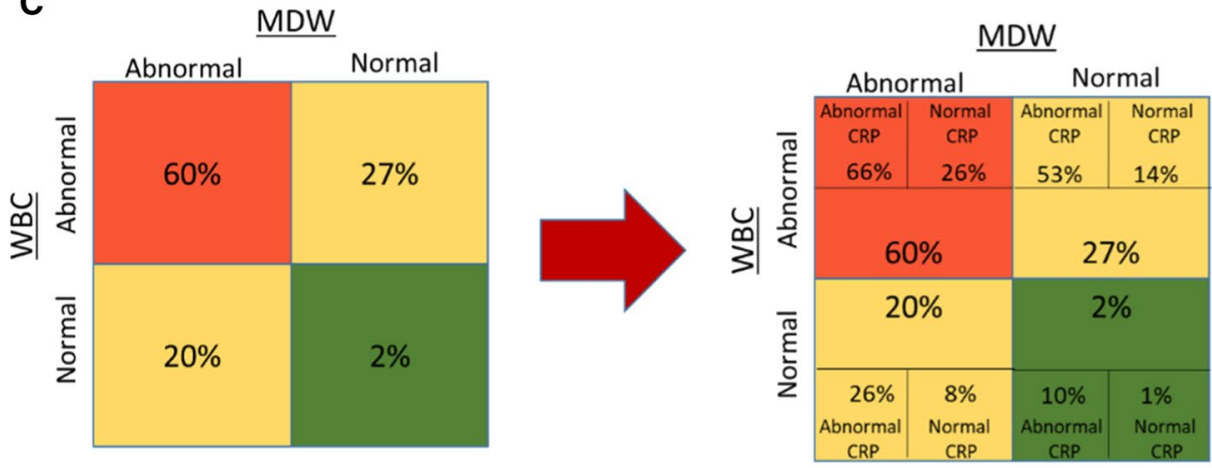

D
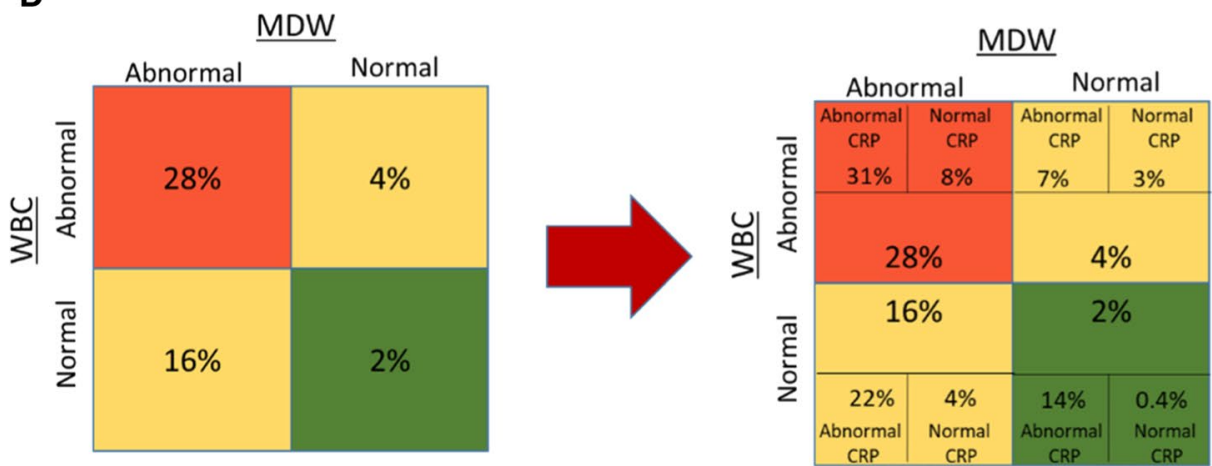

Fig. 5 Sequential assessment of sepsis probabilities according to WBC, MDW and PCT results. Cutoff: WBC $<4,000 / \mathrm{mm}^{3}$ or WBC $>12,000 / \mathrm{mm}^{3}$, MDW > 21.5, PCT > $0.25 \mu \mathrm{g} / \mathrm{L}, \mathrm{CRP}>22 \mathrm{mg} / \mathrm{L})$. Pretest probabilities were 0.17 for sepsis-2 and 0.09 for sepsis-3. A PCT by Sepsis-2. B PCT by Sepsis-3. C CRP by Sepsis-2. D CRP by Sepsis-3. MDW, monocyte distribution width; WBC, white blood count; PCT, procalcitonin; CRP, C-reactive protein 
had been reported to date in the literature, with AUCs ranging from 0.64 to 0.79 and 0.57 to 0.79 , respectively [23-27]. The first explanation of the discrepancy is that our main inclusion criterion (ED patients for whom initial evaluation included a CBC-DIFF) resulted in a less selected patient population than the criteria of previous studies (patients with a suspicion of infection or sepsis). The sepsis prevalence observed in current study (Table 1) was higher than the mean prevalence in ED population [28], but much lower than what is reported in more selected populations [23, 24]. For example, Lungström, et al., reported a Sepsis- 2 prevalence of $42 \%$ in a cohort of 1,572 EDs patients with a suspicion of infection, along with PCT and CRP AUCs of O.64 [0.61-0.67] and 0.57 [0.54-0.60], respectively, whereas Uusitalo-Seppälä, et al., reported a Sepsis- 2 prevalence of $57 \%$ and AUCs of 0.77 [0.71-0.84] and 0.60 [0.51-0.69], respectively, for severe sepsis detection $[23,24]$. The second hypothesis was that although the adjudicators were blinded to PCT and CRP results performed by protocol, they had access to PCT and CRP values obtained as standard of care, which may have contributed to an overestimation of the performance of these two sepsis biomarkers in our study.

On first analysis, our data did not support the addition of PCT and CRP testing to WBC plus MDW in the ED. However, as illustrated in Fig. 5, a sequential approach with WBC plus MDW as a systematic screening test, followed by PCT or CRP measurement in cases of discordant results (WBC abnormal-MDW normal, or WBC normal-MDW abnormal) may represent a pragmatic perspective.

We acknowledge several limitations of the study. First, as there is no gold standard for the diagnosis of sepsis, we cannot exclude the possibility of some misclassification of patients that limits or biases the accuracy of the biomarkers. However, we tried to limit this risk by adjudicating all cases using two independent adjudicators and arbitration by a third physician in case of disagreement. Second, as discussed above, the adjudicators were not blinded to PCT and CRP values ordered as standard of care, which may have contributed to an overestimation of both the prevalence of sepsis and the performance of these biomarkers. Similarly, sepsis biomarker ordering as a standard of care was different by sites (PCT and CRP in LPS, CRP only in UHG). We think that this current practice discrepancy rather enhances the validity of the combined sites results than biases it. Finally, the overall sepsis prevalence we reported was higher than the usual prevalence in EDs, arguing for a part of screening selection bias toward sepsis patients at inclusion.

\section{Conclusion}

MDW in combination with WBC has the diagnostic accuracy to detect sepsis, particularly when assessed in patients with lower pretest sepsis probability. We suggest the use of MDW as a systematic screening test, used together with SIRS criteria and qSOFA score to improve the accuracy of sepsis diagnosis $[29,30]$ in the emergency department. The place of CRP or PCT thereafter remains to be determined.

\section{Supplementary Information}

The online version contains supplementary material available at https://doi. org/10.1186/s13054-021-03622-5.

Additional file 1. Combining MDW and WBC to improve sepsis detection per Sepsis-2 and Sepsis-3 criteria. Abbreviations: MDW, monocyte distribution width; WBC, white blood count.

Additional file 2. Microbiological Tests per Sepsis-2 and Sepsis-3 Criteria.

Additional file 3. Added value of MDW to SIRS criteria for Sepsis2 diagnosis (pre-test $=0.17$ ) and to qSOFA for Sepsis-3 diagnosis (pre-test $=0.09$ ). Abbreviations: MDW, monocyte distribution width; SIRS, systemic inflammatory response syndrome; qSOFA, quick Sequential Organ Failure Assessment.

Additional file 4. Added value of MDW on sepsis post-test probabilities according to WBC range at presentation. A: Sepsis-2 (sepsis pre-test probability $=0.17$ ). B: Sepsis-3 (pre-test 0.09). C: Sepsis-2, low pre-test probability population (pre-test $=0.065$ ) $\mathrm{D}$ : low pre-test probability population per Sepsis-3 (pre-test $=0.043$ ).

\section{Acknowledgements}

The authors wish to acknowledge the support of the various clinical coordinators at UHG and LPS and the additional physician adjudicators at UHG that supported the study. LPS particularly thanks Ilaria Cherubini and Enfel Houas for excellent clinical research tasks, and Juliana Fernandes for dedicated MDW measurement. UHG also acknowledges Tania Bruno Garcia and Maite Calzado Flores for outstanding help with clinical data collection and monitoring. The authors are also grateful to the following Beckman Coulter employees Mohamad Hasan and Gang Xu, for statistical support, to Justin Rohrbach for creation of the eCRF \& data management and to Ilya Grigorov for critical review and edits to this manuscript.

\section{Authors' contributions}

Study designwas decided by PH, LT, and LV. Patient's recruitment and inclusion were performed by NRB, AMM, MC, and JP. Laboratory testing including MDW was organized and validated by MDQ, MA, and CM. Data management was performed by IC and DC. Adjudication was performed by JP, MC, NRB, and $\mathrm{AMM}$. Arbitration was performed by PH. Data analysis was completed by $\mathrm{PH}, \mathrm{LT}, \mathrm{LV}, \mathrm{MC}, \mathrm{CM}$, and NRB. Manuscript writing was performed by $\mathrm{PH}$ and LT. All authors participated in the critical review of the manuscript and approved the final version.

\section{Funding}

This study was funded by Beckman Coulter.

Availability of data and materials

On request at Beckman Coulter, Inc. 


\section{Declarations}

\section{Ethics approval and consent to participate}

The study was registered in ClinicalTrials.gov (NCT03588325) and approved by the ethical committees of the respective participating countries. Patients were included after informed consent. Study was approved by: Agence nationale de sécurité du médicament et des produits de santé (ANSM), July 02, 2018, and by the Comité de Protection des Personnes (CPP) July 16, 2018. REF. 2018A00892-53 Paris, France. Comités de Ética de la Investigación (CEI), Hospital Universitari Germans Trias i Pujol, June 22, 2018. REF.CEI PI-18-140 Badalona, Spain.

\section{Consent for publication}

NA.

\section{Competing interests}

$\mathrm{PH}$ receive consultant and advisory board fees from Beckman Coulter, educational support and congress communication fees from ThermoFisher Scientific, Radiometer and bioMérieux. IC, DC and LT are employed by Beckman Coulter, Inc., the sponsor of the clinical study. The other authors have no conflict of interest or competing interest to declare.

\section{Author details}

'Emergency Department, Hôpital Pitié-Salpêtrière, APHP-Sorbonne Université, 83 Boulevard de l'hôpital, 75651 Paris Cedex 13, France. ${ }^{2}$ Sorbonne Université, GRC-14 BIOSFAST, Paris, France. ${ }^{3}$ UMR INSERM 1166, IHU ICAN, Sorbonne Université, Paris, France. ${ }^{4}$ Emergency Department, Hospital Universitari Germans Trias I Pujol, Badalona, Spain. ${ }^{5}$ Universitat Autònoma de Barcelona, Badalona, Spain. ${ }^{6}$ Biochemisty and Emergency Biology Department, Hôpital Pitié-Salpêtrière, APHP-Sorbonne Université, Paris, France. ${ }^{7}$ Laboratory Medicine Department, Laboratori Clinic Metropolitana Nord, Hospital Universitari Germans Trias I Pujol, Badalona, Spain. ${ }^{8}$ Microbiology Department, Laboratori Clinic Metropolitana Nord, Hospital Universitari Germans Trias I Pujol, Badalona, Spain. ${ }^{9}$ Beckman Coulter, Inc., Miami, FL, USA.

Received: 17 March 2021 Accepted: 31 May 2021

Published online: 30 June 2021

\section{References}

1. Page DB, Donnelly JP, Wang HE. Community-, healthcare-, and hospitalacquired severe sepsis hospitalizations in the university healthsystem consortium. Crit Care Med. 2015;43(9):1945-51. https://doi.org/10.1097/ CCM.0000000000001164.

2. EPISEPSIS: a reappraisal of the epidemiology and outcome of severe sepsis in French intensive care units. Intens Care Med. 2004;30(4):580-588. https://doi.org/10.1007/s00134-003-2121-4

3. Leisman DE. Sepsis presenting in hospitals versus emergency departments: demographic, resuscitation, and outcome patterns in a multicenter retrospective cohort. J Hospital Med. 2019. https://doi.org/10. 12788/jhm.3188.

4. Anand V, Zhang Z, Kadri SS, Klompas M, Rhee C. Epidemiology of quick sequential organ failure assessment criteria in undifferentiated patients and association with suspected infection and sepsis. Chest 2019;156(2):289-97. https://doi.org/10.1016/j.chest.2019.03.032.

5. Rhodes A, Evans LE, Alhazzani W, et al. Surviving sepsis campaign: international guidelines for management of sepsis and septic shock: 2016. Intensive Care Med. 2017:43(3):304-77. https://doi.org/10.1007/ s00134-017-4683-6.

6. Kalil AC, Machado FR. Quick sequential organ failure assessment is not good for ruling sepsis in or out. Chest. 2019;156(2):197-9. https://doi.org/ 10.1016/j.chest.2019.06.003.

7. Pierrakos C, Velissaris D, Bisdorff M, Marshall JC, Vincent J-L. Biomarkers of sepsis: time for a reappraisal. Crit Care. 2020. https://doi.org/10.1186/ s13054-020-02993-5.
8. Crouser ED, Parrillo JE, Seymour C, et al. Improved early detection of sepsis in the ED with a novel monocyte distribution width biomarker. Chest. 2017;152(3):518-26. https://doi.org/10.1016/j.chest.2017.05.039.

9. Crouser ED, Parrillo JE, Seymour CW, et al. Monocyte distribution width: a novel indicator of sepsis-2 and sepsis-3 in high-risk emergency department patients. Crit Care Med. 2019;47(8):1018-25. https://doi.org/10. 1097/CCM.0000000000003799.

10. Crouser ED, Parrillo JE, Martin GS, et al. Monocyte distribution width enhances early sepsis detection in the emergency department beyond SIRS and qSOFA. J Intens Care. 2020. https://doi.org/10.1186/ s40560-020-00446-3.

11. Polilli E, Sozio F, Frattari A, et al. Comparison of monocyte distribution width (MDW) and procalcitonin for early recognition of sepsis. Kou YR ed. PLoS ONE. 2020;15(1):e0227300. https://doi.org/10.1371/journal.pone. 0227300

12. Levy MM, Fink MP, Marshall JC, et al. 2001 SCCM/ESICM/ACCP/ ATS/SIS international sepsis definitions conference. Crit Care Med. 2003;31(4):1250-6. https://doi.org/10.1097/01.CCM.0000050454.01978. $3 \mathrm{~B}$

13. Seymour CW, Liu VX, Iwashyna TJ, et al. Assessment of clinical criteria for sepsis: for the third international consensus definitions for sepsis and septic shock (sepsis-3). JAMA. 2016;315(8):762. https://doi.org/10.1001/ jama.2016.0288.

14. The SOFA (Sepsis-related Organ Failure Assessment) score to describe organ dysfunction/failure 4.

15. Levy MM, Fink MP, Marshall JC, et al. 2001 SCCM/ESICM/ACCP/ATS/ SIS international sepsis definitions conference. Intens Care Med. 2003;29(4):530-8. https://doi.org/10.1007/s00134-003-1662-x.

16. Shankar-Hari M, Phillips GS, Levy ML, et al. Developing a new definition and assessing new clinical criteria for septic shock: for the third international consensus definitions for sepsis and septic shock (sepsis-3). JAMA. 2016;315(8):775. https://doi.org/10.1001/jama.2016.0289.

17. Herrendörfer G, Johnson NL, Kotz S, Kemp AW. Univariate discrete distributions. 2nd ed. Wiley; 1992.

18. DeLong ER, DeLong DM, Clarke-Pearson DL. Comparing the areas under two or more correlated receiver operating characteristic curves: a nonparametric approach. Biometrics. 1988;44(3):837-45.

19. McGee S. Simplifying likelihood ratios. J Gen Intern Med. 2002;17(8):6469. https://doi.org/10.1046/j.1525-1497.2002.10750.x.

20. Deeks JJ, Altman DG. Diagnostic tests 4: likelihood ratios. BMJ. 2004;329(7458):168-9. https://doi.org/10.1136/bmj.329.7458.168.

21. Vincent J-L, Martin GS, Levy MM. qSOFA does not replace SIRS in the definition of sepsis. Crit Care. 2016. https://doi.org/10.1186/ s13054-016-1389-z.

22. Hwang SY, Jo IJ, Lee SU, et al. Low accuracy of positive qSOFA criteria for predicting 28-day mortality in critically ill septic patients during the early period after emergency department presentation. Ann Emerg Med. 2018;71(1):1-9.e2. https://doi.org/10.1016/j.annemergmed.2017.05.022.

23. Ljungström L, Pernestig A-K, Jacobsson G, Andersson R, Usener B, Tilevik D. Diagnostic accuracy of procalcitonin, neutrophil-lymphocyte count ratio, C-reactive protein, and lactate in patients with suspected bacterial sepsis. Azevedo LCP ed. PLoS ONE. 2017;12(7):e0181704. https://doi.org/ 10.1371/journal.pone.0181704.

24. Uusitalo-Seppälä R, Koskinen P, Leino A, Peuravuori H, Vahlberg T, Rintala EM. Early detection of severe sepsis in the emergency room: diagnostic value of plasma C-reactive protein, procalcitonin, and interleukin-6. Scand J Infect Dis. 2011;43(11-12):883-90. https://doi.org/10.3109/00365 548.2011 .600325

25. Claessens Y-E, Mathevon T, Kierzek G, et al. Accuracy of C-reactive protein, procalcitonin, and mid-regional pro-atrial natriuretic peptide to guide site of care of community-acquired pneumonia. Intensive Care Med. 2010;36(5):799-809. https://doi.org/10.1007/s00134-010-1818-4.

26. Hausfater P, Juillien G, Madonna-Py B, Haroche J, Bernard M, Riou B. Serum procalcitonin measurement as diagnostic and prognostic marker in febrile adult patients presenting to the emergency department. Crit Care. 2007:11(3):R60. https://doi.org/10.1186/cc5926.

27. Hausfater P, Garric S, Ayed SB, Rosenheim M, Bernard M, Riou B. Usefulness of procalcitonin as a marker of systemic infection in emergency 
department patients: a prospective study. Clin Infect Dis. 2002;34(7):895901. https://doi.org/10.1086/339198.

28. Wang HE, Jones AR, Donnelly JP. Revised national estimates of emergency department visits for sepsis in the United States. Crit Care Med. 2017:45(9):1443-9. https://doi.org/10.1097/CCM.0000000000002538.

29. Long B, April MD. Are qSOFA criteria better than the systemic inflammatory response syndrome criteria for diagnosing sepsis and predicting inhospital mortality? Ann Emerg Med. 2018;72(4):470-2. https://doi.org/ 10.1016/j.annemergmed.2018.03.008.
30. Henning DJ, Puskarich MA, Self WH, et al. An emergency department validation of the SEP-3 sepsis and septic shock definitions and comparison with 1992 consensus definitions. Ann Emerg Med. 2017;70(4):544-552.e5. https://doi.org/10.1016/j.annemergmed.2017.01.008.

\section{Publisher's Note}

Springer Nature remains neutral with regard to jurisdictional claims in published maps and institutional affiliations.
Ready to submit your research? Choose BMC and benefit from:

- fast, convenient online submission

- thorough peer review by experienced researchers in your field

- rapid publication on acceptance

- support for research data, including large and complex data types

- gold Open Access which fosters wider collaboration and increased citations

- maximum visibility for your research: over 100M website views per year

At BMC, research is always in progress.

Learn more biomedcentral.com/submissions 